\title{
Effect of Alzheimer Familial Chromosomal Mutations on the Amyloid Fibril Interaction with Different PET Tracers- Insight from Molecular Modeling Studies
}

\author{
Kanagasabai Balamurugan, ${ }^{\dagger *}$ Natarajan Arul Murugan, ${ }^{\dagger *}$ Bengt Långström, ${ }^{\S}$ Agneta \\ Nordberg $^{\perp}$ and Hans Ågren, ${ }^{\dagger \& *}$ \\ † Division of Theoretical Chemistry and Biology, School of Biotechnology, Royal Institute of \\ Technology (KTH), AlbaNova University Center, S-106 91 Stockholm, Sweden \\ $\perp$ Department of Neurobiology, Care Sciences and Society, Center for Alzheimer Research, \\ Translational Alzheimer Neurobiology, Karolinska Institute, Department of Geriatric \\ Medicine, Karolinska University Hospital, Huddinge, 14186 Stockholm, Sweden \\ $\S$ Department of Chemistry, Uppsala University, 75123 Uppsala, Sweden \\ \& Institute of Nanotechnology, Spectroscopy and Quantum Chemistry, Siberian Federal \\ University, Svobodny pr. 79, 660041 Krasnoyarsk, Russia
}

\begin{abstract}
Alzheimer's disease (AD) is the most common neurodegenerative disorder. Along with an increasing number of elderly worldwide it poses a great challenge for the society and healthcare. Although sporadic AD is the common form of $\mathrm{AD}, 2-3 \%$ of the $\mathrm{AD}$ cases are expected to be due to mutations in the beta region of the amyloid precursor protein which is referred to as autosomal dominant $\mathrm{AD}$ (ADAD). ${ }^{1}$ These mutations may cause changes in the secondary structure of the amyloid beta fibrils and may alter the fibrillization rate leading to changes in the disease development and could also affect the binding to tracers used in diagnosis. In particular, from some recent clinical studies using PET tracers for detection of fibrillar amyloids it is evident that in ADAD patients with Arctic mutation no amyloid plaque binding can be detected with ${ }^{11} \mathrm{C}$-Pittsburgh Compound B $\left({ }^{11} \mathrm{C}-\mathrm{PIB}\right)$. However, for in vitro conditions, significant binding of ${ }^{3} \mathrm{H}-\mathrm{PIB}$ has been reported for the amyloid fibrils carrying the Arctic mutation (Yamin et al. 2017). The aim of the present study is to investigate if there is any mutation specific binding of commonly used amyloid tracers, namely Florbetaben, Florbetapir, FPIB, AZD4694, AZD2184, by means of molecular modelling techniques. Other than Arctic, ADAD mutations, such as the Dutch, Italian, Iowa and Flemish mutations, are considered in this study. We report that all tracers except florbetapir show reduced binding affinity towards amyloid beta fibrils with the Arctic mutation when compared to the native type. Moreover, florbetapir is the only tracer that binds to all mutants with increased affinity when compared to the native fibril. The results obtained from these studies could increase the understanding of the structural changes, caused due by mutation and concomitant changes in the interaction pattern of the PET tracers with the mutated variants, which in turn can be useful in selecting the appropriate tracers for the diagnosis purpose as well as for designing new tracers with desirable properties.
\end{abstract}

Keywords: Alzheimer's disease, PET tracers, Autosomal dominant Alzheimer's disease, molecular dynamics simulation, docking, MM/GBSA, familial mutations.

”kanaga@kth.se, bala 86kb@yahoo.co.in, murugan@kth.se, hagren@kth.se 


\section{INTRODUCTION}

Alzheimer's disease (AD) is the most common form of dementia as well as a neurodegenerative disorder. ${ }^{2}$ The amyloid $\left(A \beta_{1-42}\right)$ deposits remain as the pathological hallmark of $\mathrm{AD}$ and the $\mathrm{A} \beta$ cascade hypothesis is widely accepted by the scientific community. ${ }^{3}$ Molecular imaging enables the identification of $\mathrm{A} \beta$ fibrils in vivo and is clinically used in the diagnosis of AD. Thus the three PET tracers ${ }^{18} \mathrm{~F}$-florbetapir, ${ }^{18} \mathrm{~F}-$ florbetaben and ${ }^{18} \mathrm{~F}$ - flutemetamol (FPIB) have been approved by the US Food and Drug Administration and the European Medical Association (EMA) for use in clinical assessment of memory impairment to exclude AD. ${ }^{4}$

Clinically, AD can be divided into early-onset (ie younger than 65 years) and lateonset (ie, older than 65 years), whereas pathologically it is characterised by the presence of amyloid $\beta$ peptide plaques and intra-neuronal tangles of hyperphosphorylated forms of the tau protein (microtubule-associated protein tau, MAPT). ${ }^{5}$ Early-onset AD accounts for about $5-10 \%$ of all AD cases among which $13 \%$ comprise the familial AD. These familial mutations can also be called autosomal dominant Alzheimer's disease (ADAD) as they are occurring via autosomal dominant inheritance. ${ }^{6}$ In addition sporadic mutations in Alzheimer's disease are also observed.

Some of the widely known ADAD mutations are the Arctic, Italian, Dutch, Iowa and Flemish variants. ${ }^{7-11}$ It has been observed from previous experimental studies that the rate of fibril formation generally increases in those familial mutations, the Flemish variant being an exception. ${ }^{12-16}$ Earlier experimental studies have also shown that the commonly occurring familial mutations only marginally affect the predominant $\beta$-sheet conformation of the $A \beta_{42}$ peptide, ${ }^{17}$ so that the basic $\beta$-sheet dominated structure of $A \beta_{42}$ is retained in the mutated systems while the kinetics, i.e the rate of fibril formation, varies among the mutant systems with respect to the wild $A \beta_{42}$. Apart from the above mentioned mutations many other types of mutations referring to the amyloid precursor protein (APP) have been observed, however, they are present in the residues preceding the $A \beta_{1-42}$ structural region and are mainly involved in the rate of formation of $A \beta_{42}$ peptides which in turn affects the aggregation and consecutive effects.

In vitro binding studies performed in autopsy brain tissue from ADAD and sporadic AD brain tissue have shown different binding properties of amyloid PET tracers such as ${ }^{3} \mathrm{H}$ PIB, ${ }^{3} \mathrm{H}$-florbetaben and ${ }^{11} \mathrm{C}$-AZD2184.$^{18}$ In vivo PET studies with ${ }^{11} \mathrm{C}$-PIB have been unable 
to demonstrate the fibrillar plaques in brain carriers of the Arctic form of early-onset AD. ${ }^{19}$ However, in vitro studies on the binding of PIB (Pittsburgh compound -B) to wild type $A \beta_{42}$ and the Arctic protofibril has also recently been published which shows that the ${ }^{3} \mathrm{H}-\mathrm{PIB}$ compound can bind to the protofibrils of Arctic $A \beta_{42 .}{ }^{20}$ The above observations illustrate the importance of understanding the interaction of commonly used PET tracers for Alzheimer's disease with the different mutant structures of $A \beta_{42}$.

Computational studies of the protein-ligand complexes have an innate advantage of obtaining molecular level information on the underlying interaction process which can be useful in interpreting the experimental observations. In order to have an atomistic picture of the interaction of PET tracers with different mutations in $A \beta_{42}$ we have carried out a modelling study using several ADAD mutations including the Arctic, Italian, Dutch, Iowa, Flemish mutations along with the native $A \beta_{42}$ using the most widely used and experimentally studied PET tracers, namely Florbetapir, Florbetaben, FPIB, AZD4694 and AZD2184. The reason for choosing these specific mutations is because in these cases the mutated residues are present in the $A \beta_{42}$ structural regions which can directly influence the interaction with the PET tracers. It is notable that the Flemish mutation is considerably different from the other mutants in several ways; their neurotoxicity is slightly reduced with respect to the native $A \beta_{42}$; their fibrillization rate is slower compared to that of the native $A \beta_{42}$; they are characterised by vascular deposition of the amyloid. We included this system in our study because this is the only mutation present in the APP structural region of $A \beta_{42}$ with considerably contrasting features with respect to native $A \beta_{42}$ in comparison to the other mutants. ${ }^{21}$ The variation in the mode of interaction between the various mutated amyloid peptides and different PET tracers depends on few important factors - one being the conformational change induced by the mutated residue in the amyloid structure, a second factor is the direct involvement of the mutated residue on the interaction with the PET tracers and a third being that the structure of the PET tracer itself is favourable to some mutations and not favourable to other mutants. Thus the molecular level understanding on the stability of these complexes may shed light on some of the earlier results observed from in-vivo and clinical studies. We recall that the chemical accuracy of the currently available force-field methods is not sufficient to distinguish compounds having binding affinity in the subnanomolar range. Free energy calculations using the free energy perturbation (FEP) approach has been reported with the standard error $0.6 \mathrm{kcal} / \mathrm{mol}$ while docking methods are associated with the range around $2.5 \mathrm{kcal} / \mathrm{mol}$ and these results are mostly system specific. ${ }^{22}$ 
The molecular mechanics energies combined with the generalized Born and surface area continuum solvation (MM/GBSA) method and with molecular dynamics simulations (MD) is known to perform better than docking but is known to sometimes underperform when compared to the FEP approach. A recent study by Wang and co-workers illustrate that $\mathrm{MM} / \mathrm{GBSA}$ is capable of capturing the experimental binding profile of various protein-ligand complexes similar in line with FEP methods despite the incorrect prediction of the binding mode for one of the ligands. ${ }^{23}$ The standard errors are known to be lower for biomolecular targets having rigid protein backbone and well defined binding sites. However, this has not been assessed in detail for targets with considerable flexibility like in the case of the amyloid beta protofibrils. Moreover, the absolute value of the binding free energy has little significance in force-field methods and so the assessment of the results is based on the relative binding free energies when compared to a reference system (here native type amyloid beta fibril). In all, the MM/GBSA results here presented may be helpful in identifying the suitability of presently used PET tracers in detecting the amyloid condition in different mutated variants of AD.

\section{RESULTS AND DISCUSSION}

\section{Docking}

The three possible core docking sites on the $A \beta_{42}$ fibril are illustrated in Figure 1 (we refer to the computational details section for further information about different core binding sites). The results of the docking carried out using the selected PET tracers onto the native and mutant variants of the $A \beta_{42}$ peptide are presented in Table 1. All five PET tracers seem to bind to all possible core binding sites except for three specific cases (AZD2184 in Italian mutation, AZD4694 and FPIB in Iowa mutation). The docking energy values indicate that the S1 site was favourable for the native and mutant peptides in most of the cases irrespective of the PET tracers. In three specific cases, i.e florbetaben in Dutch mutation, florbetaben and AZD2184 in Iowa mutation, the S2 site is found to be ambiguously more favourable than the S1 site .

We know from our previous studies that MD simulation results may vary from that of the docking results due to various factors such as solvation and protein flexibility. ${ }^{24-26}$ We therefore cross checked our result of these ambiguous systems using MD. The results for 17 nanosecond (ns) run are given in Table 2. It is found from the MD MM/GBSA results that the 
S1 site is more favourable in terms of energy and so we focussed on the $A \beta_{42}$-ligand complexes at the S1 site for the rest of the studies.

\section{Structural analysis}

The different type of $A \beta_{42}$ mutants used in this study and their details are presented in Table 3. As we are simulating the native $A \beta_{42}$ and also their mutated systems it is essential to carry out structural analysis to understand the effect of mutation on the structural aspects of $A \beta_{42}$ which can be compared with the previous experimental observations. ${ }^{17}$ Before doing the analysis, it is essential to assess whether the simulation is well equilibrated by means of a root mean square deviation (RMSD) with respect to their initial structure. The backbone RMSD of the peptide in various $A \beta_{42}$ systems with different PET tracers was calculated and presented in Figure 2. The results indicate that most of the simulated systems are well equilibrated and saturated with RMSD values around 30 ns with the exception of the Florbetapir $-\mathrm{A} \beta_{42}$ systems which are saturated around $40 \mathrm{~ns}$. This shows that the time scale of the simulations is sufficient to attain equilibration for these complex systems. In accordance with the RMSD observation we used the last $10 \mathrm{~ns}$ of the trajectory for the MM/GBSA and secondary structure analysis to obtain meaningful results.

It is well established that the backbone hydrogen bonds are responsible for retaining the beta sheet structure of the $A \beta_{42}$ peptide assembly making it essential to analyse the backbone hydrogen bonding in the simulated system to have a view about the stability of the beta aggregate. The results of the backbone H-bonding in different systems with respect to time (see Figure 3) indicate that even though there is a fluctuation in the number of backbone H-bonds they are predominately consistent (averages around 30-35) throughout the course of the simulations. This illustrates that the fibril nature of the $A \beta_{42}$ is retained for the various mutants.

The secondary structure content of the $A \beta_{42}$ in different systems for the last 10 ns of the simulation was calculated and tabulated in Table 4 . The results indicate that the beta content of $A \beta_{42}$ varies with different mutants and also with the different PET tracers. However, it is relevant to mention that the total beta content varies from a minimum of $38 \%$ to a maximum of $59 \%$. On the whole it is observed that the predominant beta fibril nature of $A \beta_{42}$ is not affected by the mutation. An earlier in-vitro study regarding the aggregation of the $\mathrm{A} \beta_{42}$ and its different mutant peptides by Annelies et al also showed that the predominant beta nature of the peptide is retained. ${ }^{16}$ Thus it is understandable that the overall beta fibril nature 
of the peptide is retained even after these mutations. As the fibril nature of $A \beta_{42}$ is an essential requirement for the interaction of PET tracers, the predominant fibril nature of the mutations indicate that their interaction with PET tracers may be retained.

\section{MM/GBSA results}

The experimental binding affinity of the PET tracers identified with the $A \beta$ fibril were collected from literature and presented in the Table 5. These values are used as a guideline to check the binding affinities calculated using the MM/GBSA approach for the native system. It is important to mention that the experimental binding affinity results are provided in ranges of values obtained from different experimental measurements. The calculated binding affinities of all the complexes are given in Table 6. The results indicate that binding energy values of various PET tracers for the native system are in the range -20 to $-28 \mathrm{kcal} / \mathrm{mol}$ explaining the nanomolar range binding affinity of these compounds. The calculated values generally reflect experimental observations with some differences that may be attributed to the use of $A \beta_{1-40}$ in some cases of the experiments. The overall general consensus in the binding pattern can be reassured from the results of the native system.

The results indicate that all the PET tracers have a favourable binding energy with the native and mutant $A \beta_{42}$ although there is some significant reduction in terms of magnitude in some specific cases. The interaction of thioflavin-t (THT)-like molecules, i.e. AZD4694 and AZD2168, with the mutant systems is weakened in all cases in comparison to the native one, except Iowa-AZD4694 where there is a slight increase of $3.4 \mathrm{kcal} / \mathrm{mol}$. It is also noticeable that the gain in energy is due to the solvation component of the system whereas the direct interactions, van der Waals and electrostatic, are not much changed. It is also notable that the AZD2184 binding to the Flemish mutant is the weakest among all the systems with a mere $8.5 \mathrm{kcal} / \mathrm{mol}$ binding energy owing to drastic reduction in terms of electrostatics and van der Waals energy. As the Flemish mutant is considerably different from other mutants in terms of their neurotoxicity and fibrillation, it is notable that we here find that the Flemish mutants in general possess weaker binding affinities to the tracers among all the studied systems. We also find that AZD4694 and AZD2184 bind to the mutant peptides satisfactorily even though there is a slight decrease in the magnitude of interaction energy except for FlemishAZD2184. The latter has been reported to bind with high affinity in vitro both in sporadic and ADAD autopsy brain tissue. ${ }^{18}$ The in vitro binding data can be interpreted in such a way that although there is a decrease in the magnitude of interaction of AZD2184 to the mutant 
peptide (except Flemish mutant) on comparing to the native system. In the case of ADAD, an increased production and deposition of amyloid plaques (except Flemish mutant) which means a larger amount of $A \beta_{42}$ is available for the PET binding that might explain why AZD2184 was found to have better binding affinity in vitro. It is also notable that the in vitro binding studies have been carried out with ADAD samples with mostly the Swedish and presenilin (PS1) mutations which cannot be compared with the different kind of structural mutations studied in this work.

The THT like molecule FPIB is illustrating similar trends for binding as that of AZD molecules. Also in case of FPIB it can be observed that the binding energy values of the mutant systems are reduced comparing to the native $A \beta_{42}$ except the Dutch mutation in which there is a favourable interaction of around $2.8 \mathrm{kcal} / \mathrm{mol}$ comparing to the native system. On the other hand, in all other mutants the reduction in the interaction energy is negligible with a maximum of $2.5 \mathrm{kcal} / \mathrm{mol}$. The results indicate that FPIB shows more or less the same kind of interaction with the native and mutant variants of $A \beta_{42}$. In vitro binding studies with ${ }^{3} \mathrm{H}-\mathrm{PIB}$ have also shown significant binding in autopsy ADAD brain tissues in agreement with the insilico results. ${ }^{18}$

The results of the MM/GBSA interaction energy of the different PET tracers under study with native and mutant variants of $A \beta_{42}$ are illustrated in Figure 4. It is observed from the data that the stilbenoid-like molecules florbetaben and florbetapir in general show a better binding affinity to the mutated systems comparing to the THT-like molecules. For florbetapir a higher binding affinity was observed with all the mutants compared to the native $A \beta_{42}$ systems where in the case of florbetaben there was a reduction in the binding affinity for the Arctic and Iowa mutations. In both the abovementioned cases the results indicate that the reduction in energy is due to the van der Waals and electrostatic components as well as solvation terms of the interacting complexes, showing that the florbetaben tracer is less favourable for detection of these specific mutations.

\section{Residuewise contributions to the binding}

The residuewise MM/GBSA energy contributions of each system are presented in Figure 5. This analysis can give insight about the role of each residue for the interaction between $A \beta_{42}$ and the PET tracer. The illustrated results indicate that the major factors ambiguously driving the favourable interaction between the complexes are the hydrophobic residues such as phenyl alanine and valine. In our previous studies we stressed the 
importance of the NMR conformation 8 of 2BEG where the phenyl alanine core have the most favourable interaction site. ${ }^{24-26}$ The results from the residue-wise break up of the MM/GBSA contribution substantiate our earlier findings: In the case of florbetaben, in addition to the phenylalanine and valine, the leucine residues make a significant contribution towards a favourable interaction. We observe from the plot that for Iowa-AZD4694 there is a stronger interaction of the phenyl alanine and valine residues compared to the native system which may explain the exceptional nature of this mutation in that it has more favourable interaction energy than the native system. There is another exceptional case: ArcticFlorbetaben shows a significant decrease in the interaction energy with respect to the native system. A corresponding decrease in the contribution of key residues, such as phenyl alanine and valine, is observed for this complex when comparing with the native system.

As we are here dealing with the mutations involving three residues, namely alanine (A) 21, glutamic acid (E) 22 and aspartic acid (D) 23, of the $A \beta_{42}$, it is important to monitor the contributions of these residues to the interaction energy. For this purpose we separately plotted the contributions of AED in the native $A \beta_{42}$ i.e residues 21-23 (for all 5 chains) in all the systems separately and presented the results in Figure 6. This gives a closer view about the effect of these mutated residues in the interaction process. In the earlier discussion we found that Dutch-FPIB has a favourable interaction which can be understood from the results for the mutated residue interaction where the three consecutive AED triads favourably interact with FPIB in comparison to the native system. Similarly, we also observed a reduction in the Arctic and Iowa mutations with florbetaben which is understood by analysing the mutated residue contributions. The plot in Figure 6 indicates that the native system has a more favourable energy contribution in terms of the 21-23 residues comparing with the Arctic and Iowa systems. In the case of Arctic-Florbetaben system, hydrophobic residues, such as phenyl alanine, valine, and the residues around point mutation site (residue 22) are contributing to the increase in total binding free energy when compared to the native system. In the case of Flemish-AZD2184, the residues around the mutation site contribute more favourably to the binding when compared to native fibril. This illustrates the predominance of the hydrophobic core residues in dictating the $A \beta_{42}$-PET interaction process. Thus it can be understandable from these results that a multitude of factors are responsible in determining the interaction process between $A \beta_{42}$ and PET tracers, some of the important ones are the conformation of the $A \beta_{42}$ peptide and the chemical nature of the ligand. In case of mutations further factors such as the conformational change induced by the mutation (which indirectly 
leads to the change in the hydrophobic core residues of the $A \beta_{42}$ ) as well as the direct interaction of the mutated residues with the PET tracer also play important roles in determining the interaction pattern of atracer with $A \beta_{42}$.

\section{Arctic mutation}

In-vitro studies of the Arctic mutation show that they are forming fibrils whereas earlier clinical studies and pathological studies have shown $A \beta$ plaques devoid of congophilic $A \beta$ cores, indicating a lack of $\beta$-pleated sheet formation. ${ }^{17,19}$ A recent detailed postmortem study of plaque pathology associated with the APParc mutation reported the presence of C- and Nterminally truncated forms of $A \beta$ that are specific for the "Arctic" plaques. ${ }^{27}$ Clinical studies show that there is a low level of C-PIB retention in the case of Arctic mutation whereas recent in-vitro studies show that H-PIB can recognise the Arctic $A \beta_{42}$ protofibril prepared invitro and oligomers. ${ }^{19}$ Thus it seems to be a difference between the Arctic $A \beta_{42}$ in the in-vitro condition and in the clinical form. ${ }^{20}$ Our simulation results go along with the in-vitro results in that the Arctic mutants can form stable fibrils. It will be important to have further details on the most dominant form of $A \beta_{42}$ present in the case of Arctic $A D$ and also its morphology under clinical condition in order to understand the peculiar behaviour of the Arctic case and appropriately understand the PET interaction.

\section{Conclusions}

The aim of the present study was to find some underlying factors for the changes in the interaction pattern of PET tracers related to mutation induced structural changes in amyloid fibrils which could be useful in selecting appropriate tracers for the diagnosis purpose and for designing new tracers with desirable binding properties. The simulation results indicate that the familial mutations such as Dutch, Italian, Arctic, Iowa and Flemish do not change the predominant fibril nature of $A \beta_{42}$ which is consistent with the previous experimental observations. The free energy values obtained for the mutated systems with the selected PET tracers show that in general the tracers can satisfactorily bind to the mutated $A \beta_{42}$ systems except for a few cases such as the AZD2184-Flemish mutant and AZD4694 in the case of the Arctic and Flemish mutations in which there is a considerable reduction in the van der Waals and electrostatic energy components between the tracer and the $A \beta_{42}$ peptide, implicating a worse compatibility of these tracers with the specific mutations. In general the stilbenoid-like molecules florbetaben and florbetapir have a better binding affinity to the mutated systems comparing with the THT-like molecules AZD4694 and AZD2184. Florbetapir is found to 
have better binding affinity with all the mutant systems under study with respect to the native system. Florbetaben is found to have better binding to the mutant systems except Arctic and Iowa cases, here also we find a significant reduction in the van der Waals and electrostatic energy components between the tracer and the $A \beta_{42}$ peptide. The hydrophobic amino acids like phenyl alanine, valine, leucine and isoleucine are in general found to play a predominant role in the $A \beta_{42}$-PET tracer interaction process. The direct interaction of the mutated residues with the PET tracers can also influence the binding affinity of the PET tracers to the mutated variants of $A \beta_{42}$. The Arctic mutation is a peculiar case where there is a series of contradicting pieces of information given by in-vitro and clinical studies regarding their fibril nature, morphology as well as $\mathrm{N}$ - and $\mathrm{C}$ - terminal truncations, thus making a further understanding on the Arctic mutation only possible from obtaining their clinically relevant structure. The results obtained from these studies can be useful in understanding the structural changes caused by mutation and concomitant changes in the interaction pattern of the PET tracers with the mutated variants which in turn can be valuable for selecting the appropriate tracers for the diagnosis purpose as well as for designing new tracers with desirable binding properties.

\section{COMPUTATIONAL DETAILS}

\section{Protein}

The structure of $A \beta_{42}$ from the protein data bank (PDB) with PDB ID 2BEG is taken as the proteins structure which is a pentamer. ${ }^{28}$ It is well established that the amyloid exist predominately in two forms $A \beta_{40}$ and $A \beta_{42}$, here we selected $A \beta_{42}$ because this form is found to play an important role in the amyloid toxicity and they are found in increased

concentrations in brains of patients with familial Alzheimer's disease. ${ }^{29}$ It is known from the previous studies that the mutations such as Arctic, Italian, Dutch, Iowa and Flemish mutations differ from the wild type amyloid peptides in terms of aggregation kinetics but predominately the beta structure is preserved. The sequence of the native $A \beta_{42}$ along with the mutated variants with the specific amino acid mutation is shown in Scheme 1 . The sites in the $A \beta_{42}$ residues which are mutated in the cases under study are shown in Figure 7. Based on the abovementioned observation the coordinates of the wild type structure are appropriately mutated with amino acid residues specific for each mutant to generate the initial structure. The $A \beta_{42}$ has multiple binding sites for the tracers where they may be classified as surface binding sites and core binding sites. In our previous studies it was shown that the core 
binding sites are predominately dictating the tracer binding affinity trends so in this study we are restricting ourselves with the core binding sites. Three possible core sites are identified where two sites are present in the first conformer NMR structure and the site specific to phenylalanine is present in eight conformers of the NMR structure. ${ }^{24-25}$

\section{Ligands}

The PET tracers such as Florbetapir, Florbetaben, FPIB, AZD4694 and AZD2184 were used as the ligands in this study. Among these five ligands the first three ligands have been approved by FDA for clinical purpose. The last two have been studied earlier with these mutant systems. The chemical structure of the ligands under study is shown in Figure 8.

\section{Docking}

The wild type $A \beta_{42}$ structure is used as present in the NMR structure whereas in the case of the mutated $A \beta_{42}$ the system is relaxed. In case of the mutated systems $A \beta_{42}$ is equilibrated in Amber 14 using FF99SBildn parameters with $12 \AA$ solvation using TIP3P parameters (counterions were added to make the system neutral) at $300 \mathrm{~K}$ and $1 \mathrm{~atm}$ pressure for $1 \mathrm{~ns} .{ }^{30-32}$ The systems were minimized using the steepest descent algorithm followed by the equilibration process in which the side chain of the mutated residues are free to relax whereas other atoms are restrained with weak force $\left(10 \mathrm{kcal} / \mathrm{mol}^{2}\right)$. The structure obtained from the equilibration process is used as the initial structure for docking in the case of mutant systems. All the possible core sites are explored for the interaction with different ligand molecules using docking. Since the amyloid protofibril does not have well defined binding sites, blind docking has been carried out using the autodock software. ${ }^{33}$ The grid box dimension has been chosen as $65 \mathrm{X} 65 \mathrm{X} 120 \AA^{3}$ with grid size of $0.375 \AA$ which is to make sure that all the core binding sites are identified. In particular, the docking has been carried out for the $1^{\text {st }}$ and $8^{\text {th }}$ conformers of amyloid protofibril and the mutant variants so that all core sites are used in the docking studies. 50 different low energy configurations for each ligand have been identified using the genetic algorithm and these configurations differ in terms of binding mode and binding pose in different binding sites. The structures of the protein-ligand complexes with the least binding energy in different binding sites were considered for further analysis.

\section{Molecular dynamics simulations}

Amber 14 package was used for the MD simulation. The low energy protein ligand docked complex is taken and are solvated with cubic box of TIP3P water molecules upto $12 \AA$ from 
their outermost coordinates. Counterions were added to make the system neutral. FF99SBildn parameters were used for the proteins and GAFF parameters were used for the ligand molecules (ESP charges using Gaussian (B3LYP/6-31G*) and Antechamber). ${ }^{34-36}$ The systems were minimized using the steepest descent algorithm followed by the equilibration using NVT and NPT ensemble 100ps each, the temperature of the system was raised from 0 to 300K during NVT equilibration phase. The solute structure was constrained with a weak force $\left(10 \mathrm{kcal} / \mathrm{mol} \AA^{2}\right)$ in the energy minimization and equilibration steps. Then the production run of 50ns in NPT ensemble was carried out for each system without any constraint. The temperature of the system was maintained at $300 \mathrm{~K}$ and pressure was maintained at 1 atm, respectively. A $12 \AA$ cutoff was set for short-range interactions, while the electrostatic interactions beyond the cutoff were computed with the particle mesh Ewald (PME) method. ${ }^{37}$ Bonds involving hydrogen atoms were constrained with the SHAKE algorithm. A time step of 2 fs was used, and the trajectory was saved every 2 ps. The trajectories were visualized using the VMD and Pymol pograms. ${ }^{38-39}$

\section{Structural Analysis}

The structural analysis of the $A \beta_{42}$ peptide from the simulated trajectory is carried out to monitor the structural changes induced in the $A \beta_{42}$ due to the mutation. The root mean square deviation (RMSD) and number of backbone hydrogen bonds were calculated with respect to time. The secondary structural population of the peptide was calculated for the last $10 \mathrm{~ns}$ of the trajectory considering the first $40 \mathrm{~ns}$ as the equilibration time as the mutant systems have to evolve.

\section{Binding Free Energy Calculations}

The python script MMPBSA.py of Amber 14 was used to do Molecular Mechanics/Generalized Born Surface Area (MM/GBSA) calculations, ${ }^{40}$ which can be used to calculate the binding free energies of ligand with native $A \beta_{42}$ and other mutant fibrils. The data from the last $10 \mathrm{~ns}$ of the MD trajectories were used for MM/GBSA calculations in the single trajectory mode; namely, the snapshots of ligand, protein, and complex were extracted from the same trajectory (once in 50 frames). This mode was efficient and has been used extensively in binding free energy calculations. ${ }^{41}$ The average values and standard errors were calculated from the results of all the extracted snapshots. The residuewise contribution of MM/GBSA was also calculated for each system. 


\section{Supporting Information:}

The individual comparative plots of RMSD and number of H-bonds of all the simulated systems are presented for clarity purpose.

\section{Acknowledgments}

The authors acknowledge support from the Swedish Foundation for Strategic Research (SSF) through the project "New imaging biomarkers in early diagnosis and treatment of Alzheimer's disease" and the support from SLL through the project "Biomolecular profiling for early diagnosis of Alzheimer's disease". This work was supported by the grants from the Swedish Infrastructure Committee (SNIC) for the projects "Multiphysics Modelling of Molecular Materials" (SNIC2016-34-43).

\section{References:}

(1) Bekris, L. M., Yu, C.-E., Bird, T. D., \& Tsuang, D. W. (2010). Genetics of Alzheimer Disease. Journal of Geriatric Psychiatry and Neurology, 23(4), 213-227.

(2) Fratiglioni, L., De Ronchi, D., and Aguero-Torres, H. (1999) Worldwide prevalence and incidence of dementia. Drugs. Aging. 15, 365-375.

(3) Hardy, J., and Selkoe, D. J. (2002)The amyloid hypothesis of alzheimer's disease: progress and problems on the road to therapeutics. Science 297, 353-356.

(4) McGeer, P. L., Kamo, H., Harrop, R., McGeer, E. G., Martin, W. R., Pate, B. D., and Li, D. K. (1986) Comparison of PET, MRI, and CT with pathology in a proven case of Alzheimer's disease. Neurology 36, 1569-1574.

(5) Braak, H., Braak, E. (1991) Neuropathological stageing of Alzheimer-related changes, Acta. Neuropathol. 82, 239-259.

(6) Campion D., Dumanchin C., Hannequin D., Dubois, B., Belliard, S., Puel, M., et al. (1999) Early-onset autosomal dominant Alzheimer disease: prevalence, genetic heterogeneity, and mutation spectrum. Am. J. Hum. Genet. 65, 664-670.

(7) Tomidokoro, Y., Rostagno, A., Neubert, T. A., Lu, Y., Rebeck, G.W., Frangione, B., Greenberg, S.M., Ghiso, J. (2010) Iowa variant of familial Alzheimer's disease: accumulation of posttranslationally modified AbetaD23N in parenchymal and cerebrovascular amyloid deposits. Am. J. Pathol. 176, 1841-1854.

(8) Maat-Schieman, M., Roos, R., van Duinen, S. (2005) Hereditary cerebral haemorrhage with amyloidosis-Dutch type. Neuropathology 25, 288-297.

(9) Bugiani, O., Giaccone, G., Rossi, G., Mangieri, M., Capobianco, R., Morbin, M., et al. (2010) Hereditary cerebral hemorrhage with amyloidosis associated with the E693K mutation of APP. Arch. Neurol. 67, 987-995.

(10) Miravalle, L., Tokuda, T., Chiarle, R., Giaccone, G., Bugiani, O., Tagliavini, F., Frangione, B., Ghiso, J. (2000) Substitutions at codon 22 of Alzheimer's abeta 
peptide induce diverse conformational changes and apoptotic effects in human cerebral endothelial cells. J. Biol. Chem. 275, 27110-27116.

(11) Kamino, K., Orr, H.T., Payami, H., Wijsman, E.M., Alonso, M.E., Pulst, S.M., et al: (1992) Linkage and mutational analysis of familial Alzheimer disease kindreds for the APP gene region. Am. J. Hum. Genet. 51, 998-1014.

(12) Nilsberth, C., Westlind-Danielsson, A., Eckman, C.B., Condron, M.M., Axelman, K., Forsell, C., et al. (2001) The 'Arctic' APP mutation (E693G) causes Alzheimer's disease by enhanced Abeta protofibril formation. Nat. Neurosci. 4, 887-893.

(13) Murakami, K., Irie, K., Morimoto, A., Ohigashi, H., Shindo, M., Nagao, M., Shimizu, T., Shirasawa, T. (2003) Neurotoxicity and physicochemical properties of Abeta mutant peptides from cerebral amyloid angiopathy: implication for the pathogenesis of cerebral amyloid angiopathy and Alzheimer's disease. J. Biol. Chem. 278, 46179-46187.

(14) Eisenhauer, P.B., Johnson, R.J., Wells, J.M., Davies, T.A., Fine, R.E. (2000) Toxicity of various amyloid beta peptide species in cultured human blood-brain barrier endothelial cells: increased toxicity of dutch-type mutant. J. Neurosci. Res. 60, 804810.

(15) Tycko, R., Sciarretta, K.L., Orgel, J.P.R.O., and Meredith, S.C., (2009) Evidence for Novel $\beta$-Sheet Structures in Iowa Mutant $\beta$-Amyloid Fibrils. Biochemistry 48, 60726084.

(16) Hendriks, L., van Duijn, C. M., Cras, P., Cruts, M., Hul, W. V., van Harskamp, F., Warren, A., McInnis, M. G., Antonarakis, S. E., and Martin, J. J. (1992) Presenile dementia and cerebral haemorrhage linked to a mutation at codon 692 of the $\beta$ amyloid precursor protein gene. Nat. Genet. 1, 218-221.

(17) Annelies, V., Ellen, H., Rabia, S., Greet, D.B., Joost, S., Frederic, R., Vinod, S., Vincent, R., Holger, W., Dirk, W., and Kerensa, B. (2012), A comparative analysis of the aggregation behavior of amyloid- $\beta$ peptide variants, FEBS Letters 586, 40884093.

(18) Ni, R., Gillberg, P.G., Bogdanovic, N., Viitanen, M., Myllykangas, L., Nennesmo, I., Långström, B., Nordberg, A. (2017) Amyloid tracers binding sites in autosomal dominant and sporadic Alzheimer's disease, Alzheimers Dement, 13, 419-430.

(19) Scholl, M., Wall, A., Thordardottir, S., Ferreira, D., Bogdanovic, N., Langstrom, B., et al. (2012) Low PiB PET retention in presence of pathologic CSF biomarkers in Arctic APP mutation carriers. Neurology, 79,229-236.

(20) Yamin, G. and Teplow, D. B. (2017) Pittsburgh Compound-B (PiB) binds amyloid $\beta$ protein protofibrils. J. Neurochem., 140, 210-215.

(21) Murakami, K., Irie, K., Morimoto, A., Ohigashi, H., Shindo, M., Nagao, M., Shimizu, T., and Shirasawa, T. (2003) Neurotoxicity and physicochemical properties of $A \beta$ mutant peptides from cerebral amyloid angiopathy: implication for the pathogenesis of cerebral amyloid angiopathy and Alzheimer's disease. J. Biol. Chem. 278, 46179-46187.

(22) Aldeghi, M., Heifetz, A., Bodkin, M.J., Knapp, S., Biggin, P.C. (2016) Accurate calculation of the absolute free energy of binding for drug molecules. Chem Sci. 7,207-218. 
(23) Kaus, W.J., Harder, E., Lin, T., Abel, R., McCammon, J. A., and Wang, L. (2015) How to deal with multiple binding poses in alchemical relative protein-ligand binding free energy calculations. J. Chem. Theory. Comput. 11, 2670-2679.

(24) Kuang, G., Murugan, N. A., Tu, Y., Nordberg, A., and Ågren, H. (2015) Investigation of the binding profiles of AZD2184 and thioflavin T with Amyloid$\beta(1-42)$ fibril by molecular docking and molecular dynamics methods. J. Phys. Chem. B 119, 11560-11567.

(25) Murugan, N. A., Halldin, C., Nordberg, A., Långström, B., and Ågren, H. (2016) The culprit is in the cave: the core sites explain the binding profiles of amyloidspecific tracers. J. Phys. Chem. Lett. 7, 3313-3321.

(26) Balamurugan, K., Murugan, N.A., and Ågren, H. (2016) Multistep modeling strategy to improve the binding affinity prediction of PET tracers to A $\beta 42$ : case study with styrylbenzoxazole derivatives. ACS Chemical Neuroscience 7, 1698-1705.

(27) Philipson, O., Lord, A., Lalowski, M., Soliymani, R., Baumann, M., Thyberg, J., et al. The Arctic A $\beta P P$ mutation results in distinct plaques and accumulation of $\mathrm{N}$ - and C-truncated A $\beta$. Neurobiol Aging, 33, 1010.

(28) Luhrs, T., Ritter, C., Adrian, M., Riek-Loher, D., Bohrmann, B., Dobeli, H., Schubert, D., and Riek, R. (2005) 3D structure of Alzheimer's amyloid- $\beta(1-42)$ fibrils. Proc. Natl. Acad. Sci. U. S. A. 102, 17342-17347.

(29) S.G. Younkin. (1998)The role of A beta 42 in Alzheimer's disease. J Physiol Paris, $92,289-292$

(30) Case, D. A., Darden, T. A., Cheatham, T. E., III, Simmerling, C. L., Wang, J., Duke, R. E., Luo, R., Walker, R. C., Zhang, W., Merz, K. M., Roberts, B., Hayik, S., Roitberg, A., Seabra, J., Swails, A. W., Götz, I., Kolossváry, K. F., Wong, F., Paesani, J., Vanicek, R. M., Wolf, G., Liu, J., Wu, X., Brozell, S. R., Steinbrecher, T., Gohlke, H., Cai, Q., Ye, X., Wang, J., Hsieh, M.-J., Cui, G., Roe, D. R., Mathews, D. H., Seetin, M. G., Salomon-Ferrer, R., Sagui, C., Babin, V., Luchko, T., Gusarov, S., Kovalenko, A., and Kollman, P. A. (2012) AMBER 12, University of California, San Francisco, CA.

(31) Lindorff-Larsen, K., Piana, S., Palmo, K., Maragakis, P., Klepeis, J. L., Dror, R. O., \& Shaw, D. E. (2010). Improved side-chain torsion potentials for the Amber ff99SB protein force field. Proteins 78, 1950-1958.

(32) Jorgensen, W. L., Chandrasekhar, J., Madura, J. D., Impey, R. W. and Klein, M. L. (1983) Comparison of simple potential functions for simulating liquid water. $J$. Chem. Phys., 79,926-935.

(33) Morris, G. M., Huey, R., Lindstrom, W., Sanner, M. F., Belew, R. K., Goodsell, D. S., and Olson, A. J. (2009) Autodock4 and AutoDockTools4: automated docking with selective receptor flexiblity. J. Comput. Chem. 30, 2785-2791.

(34) Wang, J., Wolf, R. M., Caldwell, J. W., Kollman, P. A., and Case, D. A. (2004) Development and testing of a general amber force field. J. Comput. Chem. 25, 1157-1174.

(35) Frisch, M. J., Trucks, G. W., Schlegel, H. B., Scuseria, G. E., Robb, M. A., Cheeseman, J. R., Scalmani, G., Barone, V., Mennucci, B., Petersson, G. A., Nakatsuji, H., Caricato, M., Li, X., Hratchian, H. P., Izmaylov, A. F., Bloino, J., Zheng, G., Sonnenberg, J. L., Hada, M., Ehara, M., Toyota, K., Fukuda, R., 
Hasegawa, J., Ishida, M., Nakajima, T., Honda, Y., Kitao, O., Nakai, H., Vreven, T., Montgomery, J. A., Jr., Peralta, J. E., Ogliaro, F., Bearpark, M., Heyd, J. J., Brothers, E., Kudin, K. N., Staroverov, V. N., Kobayashi, R., Normand, J., Raghavachari, K., Rendell, A., Burant, J. C., Iyengar, S. S., Tomasi, J., Cossi, M., Rega, N., Millam, J. M., Klene, M., Knox, J. E., Cross, J. B., Bakken, V., Adamo, C., Jaramillo, J., Gomperts, R., Stratmann, R. E., Yazyev, O., Austin, A. J., Cammi, R., Pomelli, C., Ochterski, J. W., Martin, R. L., Morokuma, K., Zakrzewski, V. G., Voth, G. A., Salvador, P., Dannenberg, J. J., Dapprich, S., Daniels, A. D., Farkas, Ö., Foresman, J. B., Ortiz, J. V., Cioslowski, J., and Fox, D. J. (2009) Gaussian 09, revision D.01, Gaussian, Inc., Wallingford, CT.

(36) Wang, J., Wang, W., Kollman, P. A., and Case, D. A. (2006) Automatic atom type and bond type perception in molecular mechanical calculations. J. Mol. Graphics Modell. 25, 247-260.

(37) Essmann, U., Perera, L., Berkowitz, M. L., Darden, T., Lee, H., and Pedersen, L. G. A. (1995) Smooth particle mesh ewald method. J. Chem. Phys. 103, 8577-8593.

(38) Humphrey, W., Dalke, A., and Schulten, K. (1996) VMD: Visual Molecular Dynamics. J. Mol. Graphics 14, 33-38.

(39) The PyMOL Molecular Graphics System, version 1.3, Schrödinger, LLC.

(40) Miller, B. R., McGee, T. D., Swails, J. M., Homeyer, N., Gohlke, H., and Roitberg, A. E. (2012) MMPBSA.py: An efficient program for end-state free energy calculations. J. Chem. Theory Comput. 8, 3314-3321.

(41) Hou, T. J., Wang, J. M., Li, Y. Y., and Wang, W. (2011) Assessing the performance of the MM/PBSA and MM/GBSA Methods. 1. The accuracy of binding free energy calculations based on molecular dynamics simulations. J. Chem. Inf. Model. 51, 69-82.

(42) Kassler, K., Horn, A.H.C., and Sticht, H. (2010) Effect of pathogenic mutations on the structure and dynamics of Alzheimer's A 342 -amyloid oligomers. J. Mol. Model. $16,1011-1020$.

(43) Van Nostrand, W.E., Melchor, J.P., Cho, H.S., Greenberg, S.M., and Rebeck, G.W. (2001) Pathogenic effects of D23N Iowa mutant amyloid $\beta$-protein. J. Biol. Chem.276, 32860-32866.

(44) Ni, R., Gillberg, P.-G., Bergfors, A., Marutle, A., Nordberg, A. (2013) Amyloid tracers detect multiple binding sites in alzheimers disease brain tissue. Brain, 136, 2217-2227.

(45) Johnson, A. E., Jeppsson, F., Sandell, J., Wensbo, D., Neelissen, J. A., Jureus, A., Ström, P., Norman, H., Farde, L., Svensson, S. P. (2009) AZD2184: a radioligand for sensitive detection of $\beta$-amyloid deposits. J. Neurochem., 108, 1177-1186.

Table 1. The results of various PET tracers docked with the core binding sites of the wild type and mutant $A \beta_{42}$ systems (energy in $\mathrm{kcal} / \mathrm{mol}$ ). 


\begin{tabular}{|c|c|c|c|c|}
\hline S.No & Tracer & S1 & S2 & S3 \\
\hline & Native & & & \\
\hline 1 & Florbetaben & -8.14 & -8.01 & -7.74 \\
\hline 2 & Florbetapir & -7.90 & -7.68 & -7.45 \\
\hline 3 & FPIB & -8.66 & -8.13 & -7.05 \\
\hline 4 & AZD4694 & -8.62 & -8.04 & -7.48 \\
\hline \multirow[t]{2}{*}{5} & AZD2184 & -8.44 & -7.97 & -6.82 \\
\hline & Arctic & & & \\
\hline 6 & Florbetaben & -8.05 & -7.54 & -7.46 \\
\hline 7 & Florbetapir & -7.85 & -7.54 & -7.51 \\
\hline 8 & FPIB & -8.62 & -8.19 & -6.83 \\
\hline 9 & AZD4694 & -8.60 & -7.96 & -7.22 \\
\hline \multirow[t]{2}{*}{10} & AZD2184 & -8.40 & -8.17 & -6.56 \\
\hline & Italian & & & \\
\hline 11 & Florbetaben & -7.84 & -7.71 & -6.67 \\
\hline 12 & Florbetapir & -7.40 & -6.99 & -6.92 \\
\hline 13 & FPIB & -8.34 & -7.90 & -5.82 \\
\hline 14 & AZD4694 & -8.32 & -8.30 & -6.51 \\
\hline \multirow[t]{2}{*}{15} & AZD2184 & -8.22 & -8.18 & - \\
\hline & Dutch & & & \\
\hline 16 & Florbetaben & -7.07 & -7.33 & -7.07 \\
\hline 17 & Florbetapir & -7.04 & -7.01 & -6.87 \\
\hline 18 & FPIB & -8.25 & -7.78 & -6.69 \\
\hline 19 & AZD4694 & -8.37 & -7.74 & -7.41 \\
\hline \multirow[t]{2}{*}{20} & AZD2184 & -8.11 & -7.72 & -6.93 \\
\hline & Iowa & & & \\
\hline 21 & Florbetaben & -7.37 & -7.67 & -5.89 \\
\hline 22 & Florbetapir & -7.33 & -7.03 & -6.07 \\
\hline 23 & FPIB & -8.29 & -7.87 & - \\
\hline 24 & AZD4694 & -8.45 & -7.99 & - \\
\hline \multirow[t]{2}{*}{25} & AZD2184 & -7.99 & -8.20 & -7.26 \\
\hline & Flemish & & & \\
\hline 26 & Florbetaben & -8.39 & -6.94 & -7.91 \\
\hline 27 & Florbetapir & -9.34 & -7.85 & -8.58 \\
\hline 28 & FPIB & -8.31 & -7.95 & -7.66 \\
\hline 29 & AZD4694 & -8.11 & -8.22 & -7.61 \\
\hline 30 & AZD2184 & -8.86 & -8.15 & -8.16 \\
\hline
\end{tabular}

Table 2. MM/GBSA calculated binding affinities ( $\left.\Delta \mathrm{G}_{\text {bind }}\right)$ of selected system at the S1 and S2 binding sites (energy in $\mathrm{kcal} / \mathrm{mol}$ ). 


\begin{tabular}{|c|l|c|c|}
\hline S.No & \multicolumn{1}{|c|}{ Tracer } & S1 & S2 \\
\hline & Dutch & & \\
\hline 1 & Florbetaben & -23.65 & -20.99 \\
\hline & Iowa & & \\
\hline 2 & Florbetaben & -23.37 & -16.89 \\
\hline 3 & AZD2184 & -18.27 & -16.49 \\
\hline
\end{tabular}

Table 3. The different types of $A \beta_{42}$ mutants, mutations, neurotoxicity and their fibrillation rate. $^{42,43}$

\begin{tabular}{|c|c|c|c|c|}
\hline S.No & Name & Mutation & Neurotoxicity & Rate \\
\hline 1 & Native & - & - & - \\
\hline 2 & Arctic mutation & E22G & Enhanced & Accelerated \\
\hline 3 & Italian mutation & E22K & Enhanced & Accelerated \\
\hline 4 & Dutch mutation & E22Q & Enhanced & Accelerated \\
\hline 5 & Iowa mutation & D23N & Enhanced & Accelerated \\
\hline 6 & Flemish mutation & A21G & Slightly weakened & Decreased \\
\hline
\end{tabular}

Table 4. Beta sheet content of different PET-A $\beta_{42}$ systems simulated.

\begin{tabular}{|c|c|c|c|c|c|c|}
\hline S.No & System & Florbetaben & Florbetapir & FPIB & AZD4694 & AZD2184 \\
\hline 1 & Native & 48.08 & 55.21 & 46.66 & 43.90 & 41.50 \\
\hline 2 & Dutch & 48.70 & 51.54 & 53.82 & 45.79 & 46.11 \\
\hline 3 & Italian & 45.18 & 43.57 & 45.83 & 40.81 & 37.56 \\
\hline 4 & Arctic & 48.22 & 43.87 & 59.15 & 44.90 & 49.47 \\
\hline 5 & Iowa & 45.15 & 40.07 & 40.74 & 47.27 & 48.04 \\
\hline 6 & Flemish & 50.86 & 50.34 & 42.48 & 52.36 & 51.77 \\
\hline
\end{tabular}

Table 5. Experimental binding affinities of various PET tracers used in this study. ${ }^{25,44-45}$

\begin{tabular}{|c|c|c|c|}
\hline S.No & PET tracer & Target & $k_{i}$ \\
\hline 1 & Flnrhetahen & $\mathrm{AR}$ & ว9-3 $\mathrm{nM}$ \\
\hline
\end{tabular}

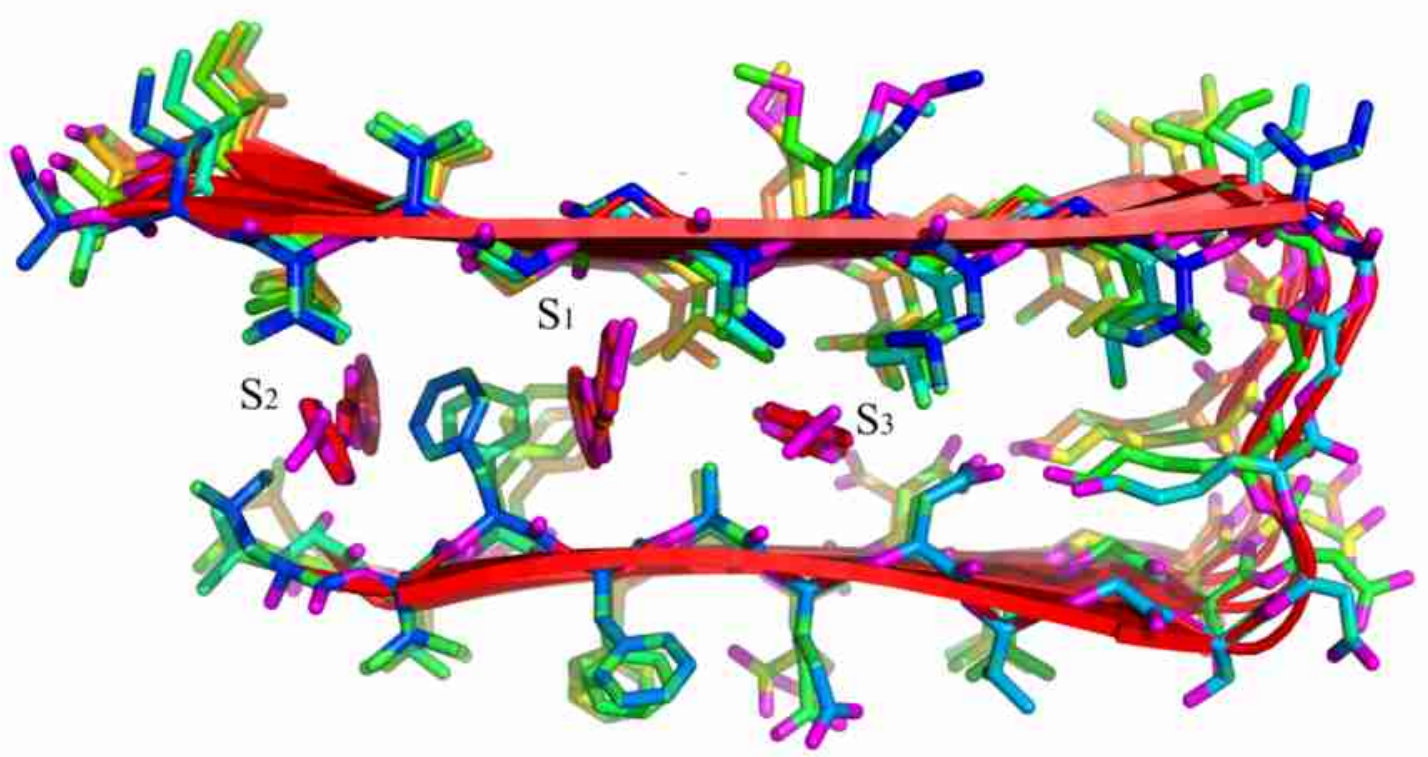


Figure 1. Schematic representation of the three possible core binding sites in the $A \beta_{42}$ peptide.

Table 6. MM/GBSA calculated binding affinities of different PET-A $\beta_{42}$ complexes (energy in $\mathrm{kcal} / \mathrm{mol})$.

\begin{tabular}{|l|l|l|l|l|l|l|l|c|}
\hline S.No & System & $\Delta \mathrm{E}_{\mathrm{Vdw}}$ & $\Delta \mathrm{E}_{\text {elec }}$ & $\Delta \mathrm{G}_{\mathrm{GB}}$ & $\Delta \mathrm{G}_{\mathrm{SASA}}$ & $\mathrm{T} \Delta \mathrm{S}$ & $\Delta \mathrm{G}_{\text {bind }}$ & $\Delta \Delta \mathrm{G}_{\text {bind }}$ \\
\hline & Native & & & & & & & \\
\hline 1 & Florbetaben & $-56.01 \pm 0.24$ & $-15.51 \pm 0.40$ & $27.99 \pm 0.38$ & $-7.80 \pm 0.02$ & $-23.44 \pm 0.52$ & -27.91 & - \\
\hline 2 & Florbetapir & $-50.36 \pm 0.35$ & $-3.64 \pm 0.20$ & $15.48 \pm 0.19$ & $-6.54 \pm 0.04$ & $-24.00 \pm 0.52$ & -21.06 & - \\
\hline
\end{tabular}




\begin{tabular}{|c|c|c|c|c|c|c|c|c|}
\hline 3 & FPIB & $-46.69 \pm 0.22$ & $-5.81 \pm 0.38$ & $15.81 \pm 0.31$ & $-4.90 \pm 0.01$ & $-21.12 \pm 0.47$ & -20.48 & - \\
\hline 4 & AZD4694 & $-41.87 \pm 0.23$ & $-15.12 \pm 0.36$ & $24.48 \pm 0.31$ & $-4.62 \pm 0.01$ & $-17.13 \pm 0.52$ & -20.00 & - \\
\hline \multirow[t]{2}{*}{5} & AZD2184 & $-45.57 \pm 0.22$ & $-17.70 \pm 0.27$ & $25.91 \pm 0.18$ & $-4.34 \pm 0.01$ & $-17.91 \pm 0.52$ & -23.80 & - \\
\hline & Arctic & & & & & & & \\
\hline 6 & Florbetaben & $-46.40 \pm 0.07$ & $-7.12 \pm 0.08$ & $15.36 \pm 0.05$ & $-6.26 \pm 0.01$ & $-25.63 \pm 0.37$ & -18.80 & 9.11 \\
\hline 7 & Florbetapir & $-58.41 \pm 0.36$ & $-11.11 \pm 0.34$ & $22.74 \pm 0.33$ & $-6.82 \pm 0.03$ & $-26.65 \pm 0.45$ & -26.94 & -5.88 \\
\hline 8 & FPIB & $-45.33 \pm 0.07$ & $-4.89 \pm 0.12$ & $16.41 \pm 0.12$ & $-4.88 \pm 0.01$ & $-19.70 \pm 0.38$ & -19.00 & 1.48 \\
\hline 9 & AZD4694 & $-36.81 \pm 0.06$ & $-2.99 \pm 0.09$ & $12.72 \pm 0.08$ & $-4.16 \pm 0.01$ & $-17.85 \pm 0.04$ & -13.40 & 6.60 \\
\hline \multirow[t]{2}{*}{10} & AZD2184 & $-41.99 \pm 0.21$ & $-10.21 \pm 0.28$ & $18.26 \pm 0.21$ & $-4.94 \pm 0.01$ & $-18.64 \pm 0.23$ & -20.24 & 3.56 \\
\hline & Italian & & & & & & & \\
\hline 11 & Florbetaben & $-61.92 \pm 0.08$ & $-6.19 \pm 0.11$ & $17.93 \pm 0.09$ & $-7.50 \pm 0.01$ & $-25.10 \pm 0.57$ & -32.58 & -4.67 \\
\hline 12 & Florbetapir & $-58.80 \pm 0.34$ & $-16.37 \pm 0.39$ & $24.61 \pm 0.28$ & $-6.95 \pm 0.01$ & $-23.01 \pm 0.52$ & -33.71 & -12.65 \\
\hline 13 & FPIB & $-41.64 \pm 0.07$ & $-10.98 \pm 0.07$ & $18.93 \pm 0.05$ & $-4.89 \pm 0.00$ & $-18.71 \pm 0.43$ & -19.87 & 0.61 \\
\hline 14 & AZD4694 & $-42.23 \pm 0.08$ & $-6.31 \pm 0.13$ & $15.52 \pm 0.08$ & $-4.49 \pm 0.00$ & $-20.06 \pm 0.45$ & -17.45 & 2.55 \\
\hline \multirow[t]{2}{*}{15} & AZD2184 & $-42.84 \pm 0.23$ & $-17.93 \pm 0.22$ & $25.34 \pm 0.19$ & $-4.53 \pm 0.01$ & $-17.61 \pm 0.63$ & -22.35 & 1.45 \\
\hline & Dutch & & & & & & & \\
\hline 16 & Florbetaben & $-56.16 \pm 0.11$ & $-9.15 \pm 0.09$ & $19.16 \pm 0.08$ & $-7.06 \pm 0.01$ & $-24.99 \pm 0.49$ & -28.22 & -0.31 \\
\hline 17 & Florbetapir & $-63.84 \pm 0.29$ & $-4.93 \pm 0.18$ & $15.48 \pm 0.23$ & $-7.99 \pm 0.01$ & $-26.25 \pm 0.42$ & -35.04 & -13.98 \\
\hline 18 & FPIB & $-42.57 \pm 0.06$ & $-5.88 \pm 0.06$ & $14.34 \pm 0.05$ & $-4.86 \pm 0.00$ & $-15.74 \pm 0.50$ & -23.23 & -2.75 \\
\hline 19 & AZD4694 & $-39.64 \pm 0.06$ & $-8.56 \pm 0.10$ & $17.58 \pm 0.07$ & $-4.42 \pm 0.00$ & $-18.71 \pm 0.31$ & -16.34 & 3.66 \\
\hline \multirow[t]{2}{*}{20} & AZD2184 & $-41.01 \pm 0.22$ & $-10.44 \pm 0.20$ & $17.90 \pm 0.13$ & $-4.61 \pm 0.01$ & $-20.78 \pm 0.46$ & -17.38 & 6.42 \\
\hline & lowa & & & & & & & \\
\hline 21 & Florbetaben & $-51.23 \pm 0.10$ & $-10.69 \pm 0.08$ & $19.13 \pm 0.06$ & $-6.61 \pm 0.01$ & $-24.59 \pm 0.36$ & -24.81 & 3.10 \\
\hline 22 & Florbetapir & $-54.43 \pm 0.31$ & $-14.90 \pm 0.39$ & $25.16 \pm 0.28$ & $-7.20 \pm 0.02$ & $-25.94 \pm 0.54$ & -25.44 & -4.38 \\
\hline 23 & FPIB & $-41.09 \pm 0.07$ & $-15.96 \pm 009$ & $23.04 \pm 0.05$ & $-4.84 \pm 0.00$ & $-19.94 \pm 0.43$ & -18.92 & 1.56 \\
\hline 24 & AZD4694 & $-40.28 \pm 0.07$ & $-15.19 \pm 0.11$ & $18.60 \pm 0.05$ & $-4.73 \pm 0.11$ & $-18.24 \pm 0.53$ & -23.37 & -3.37 \\
\hline \multirow[t]{2}{*}{25} & AZD2184 & $-45.38 \pm 0.26$ & $-11.10 \pm 0.40$ & $22.88 \pm 0.30$ & $-4.77 \pm 0.01$ & $-18.86 \pm 0.48$ & -19.52 & 4.28 \\
\hline & Flemish & & & & & & & \\
\hline 26 & Florbetaben & $-51.28 \pm 0.10$ & $-16.00 \pm 0.15$ & $23.51 \pm 0.12$ & $-7.33 \pm 0.01$ & $-24.66 \pm 0.49$ & -26.44 & 1.47 \\
\hline 27 & Florbetapir & $-48.76 \pm 0.32$ & $-12.77 \pm 0.34$ & $24.64 \pm 0.33$ & $-6.27 \pm 0.03$ & $-20.38 \pm 0.62$ & -22.78 & -1.72 \\
\hline 28 & FPIB & $-42.60 \pm 0.09$ & $-6.36 \pm 0.15$ & $19.41 \pm 0.16$ & $-4.89 \pm 0.01$ & $-16.47 \pm 0.50$ & -17.98 & 2.50 \\
\hline 29 & AZD4694 & $-36.35 \pm 0.12$ & $-3.68 \pm 0.08$ & $11.90 \pm 007$ & $-4.34 \pm 0.00$ & $-17.72 \pm 0.64$ & -14.76 & 5.24 \\
\hline 30 & AZD2184 & $-34.06 \pm 0.19$ & $-3.05 \pm 0.26$ & $13.58 \pm 0.19$ & $-3.85 \pm 0.02$ & $-18.88 \pm 0.30$ & -8.51 & 15.29 \\
\hline
\end{tabular}



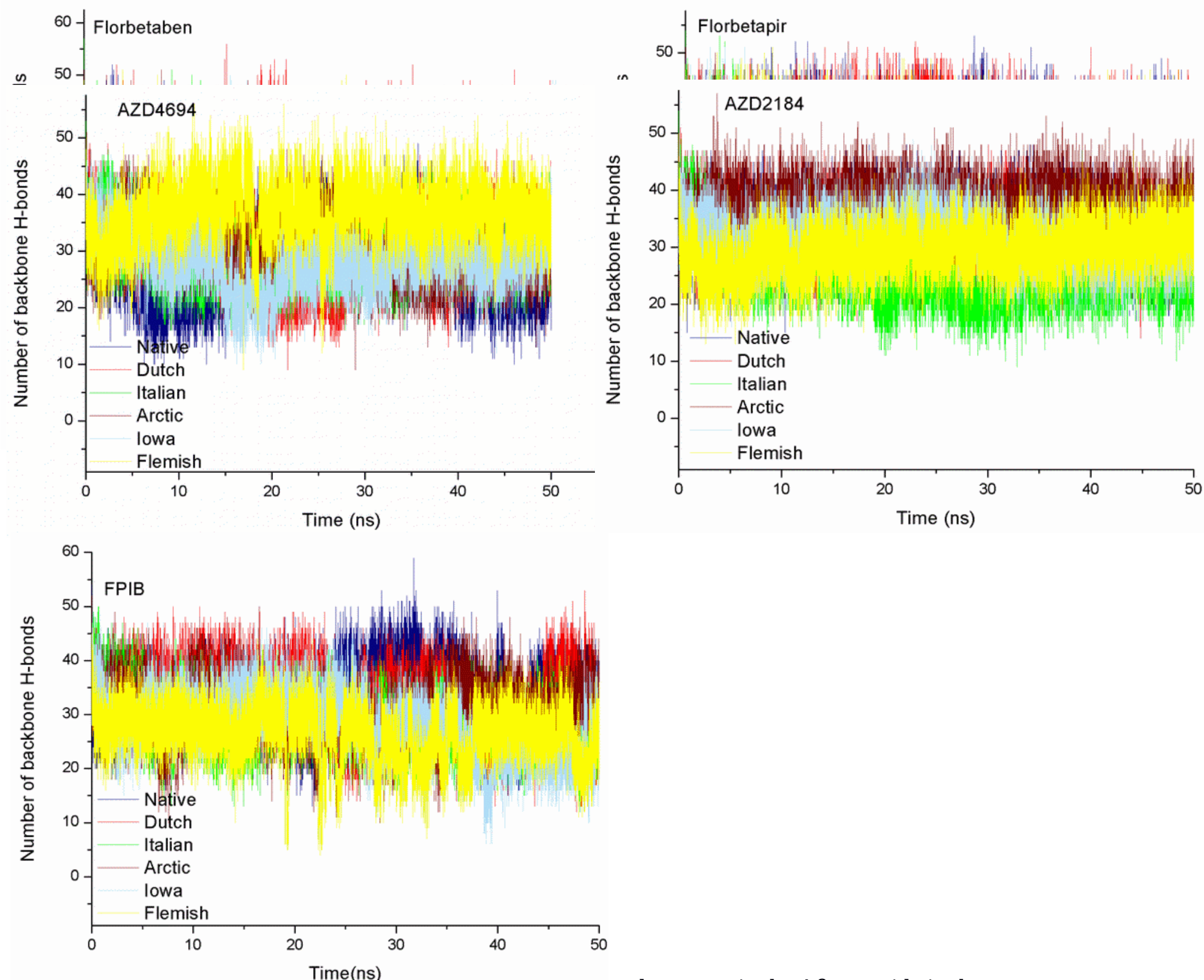

rigure s. 1 ne number oI Dack done nyarogen oonds present in the $A \beta_{42}$ peptide in the different $A \beta_{42}$-PET tracer systems simulated. 


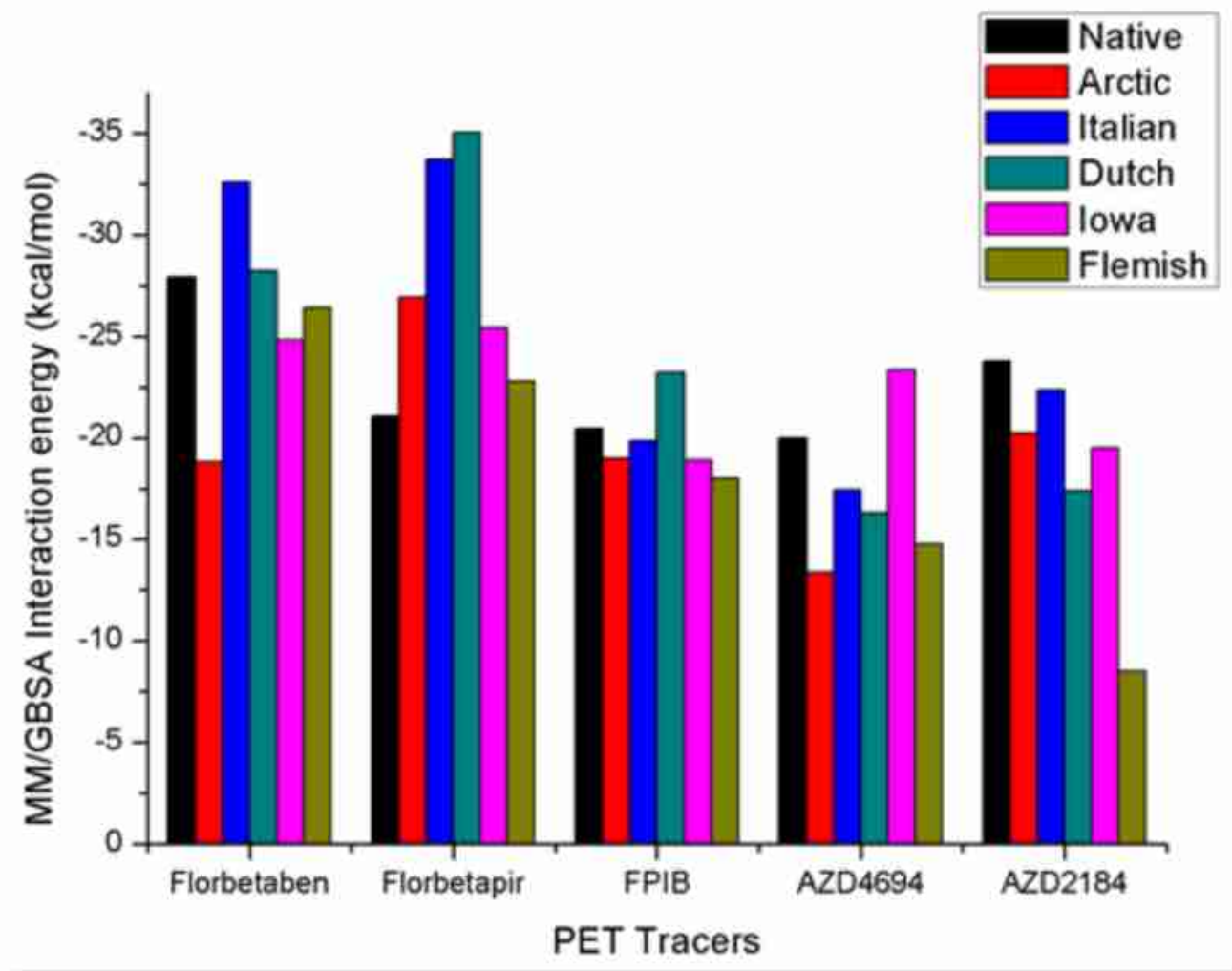

Figure 4. MM/GBSA interaction energy plot of different PET tracers with the native and mutated $A \beta_{42}$ system. 

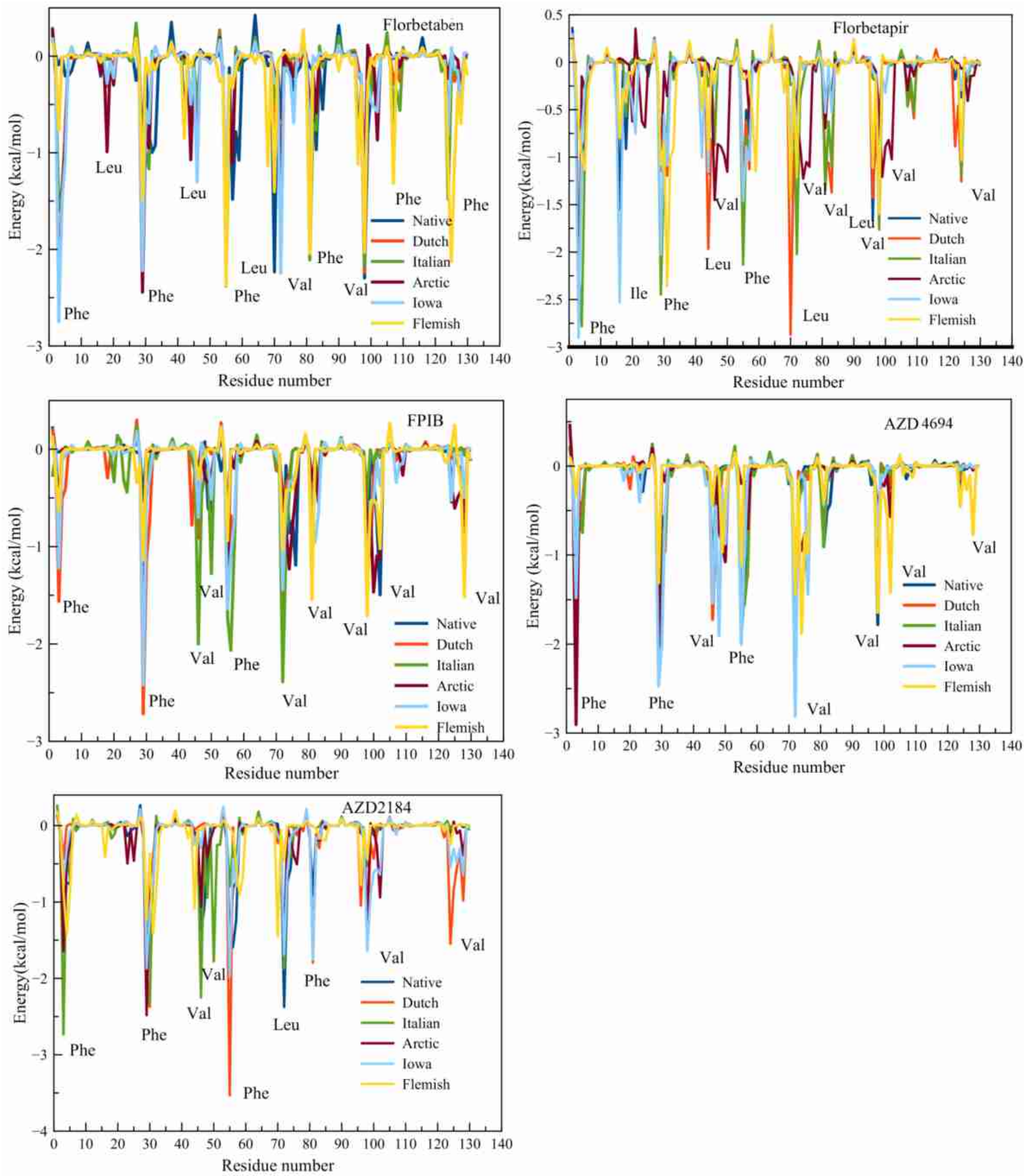

Figure 5. The residue wise contribution of the amino acid present in the $A \beta_{42}$ system towards the MM/GBSA binding free energy in the different $\mathrm{A} \beta_{42}$-PET tracer systems. 

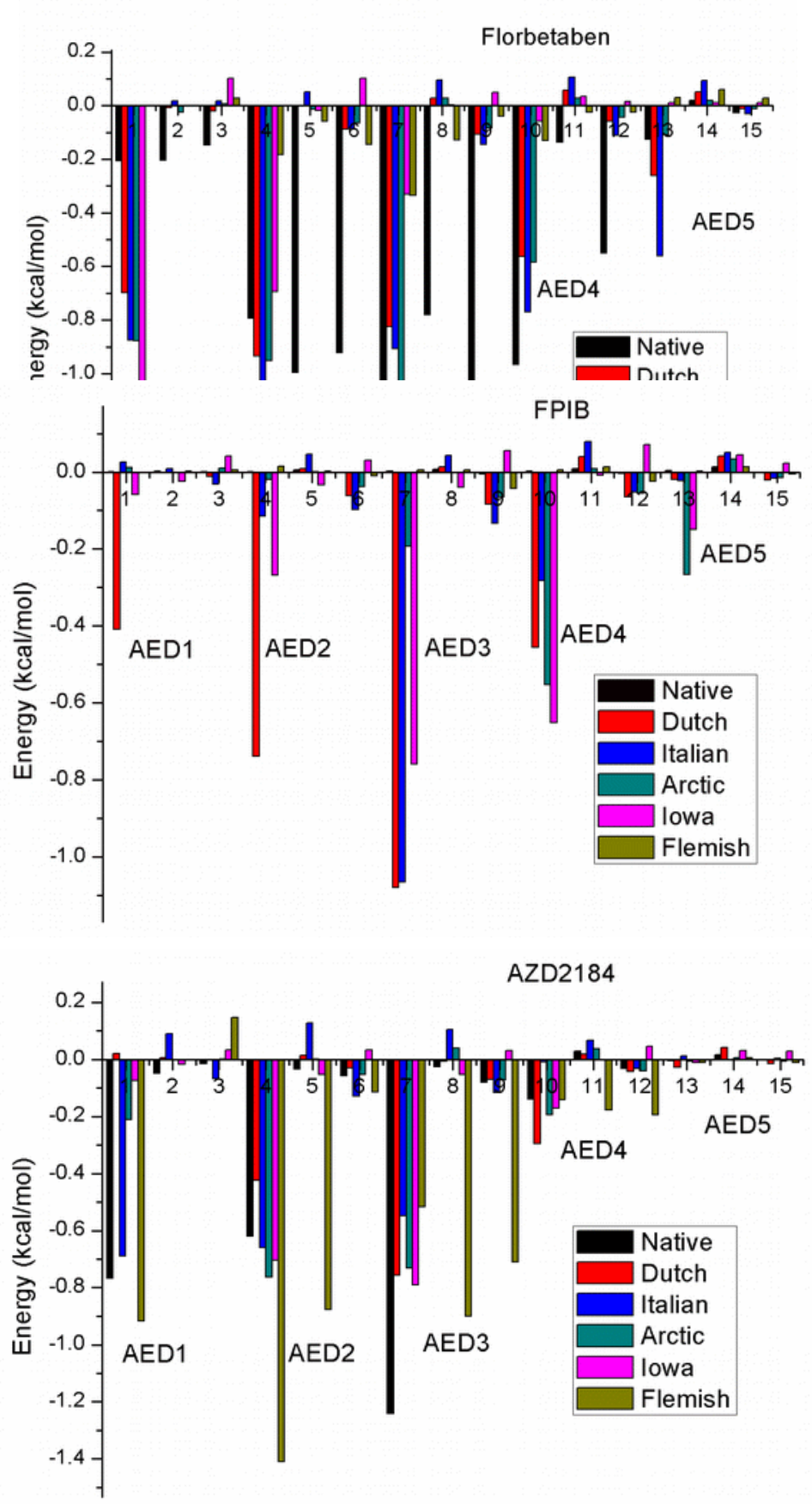

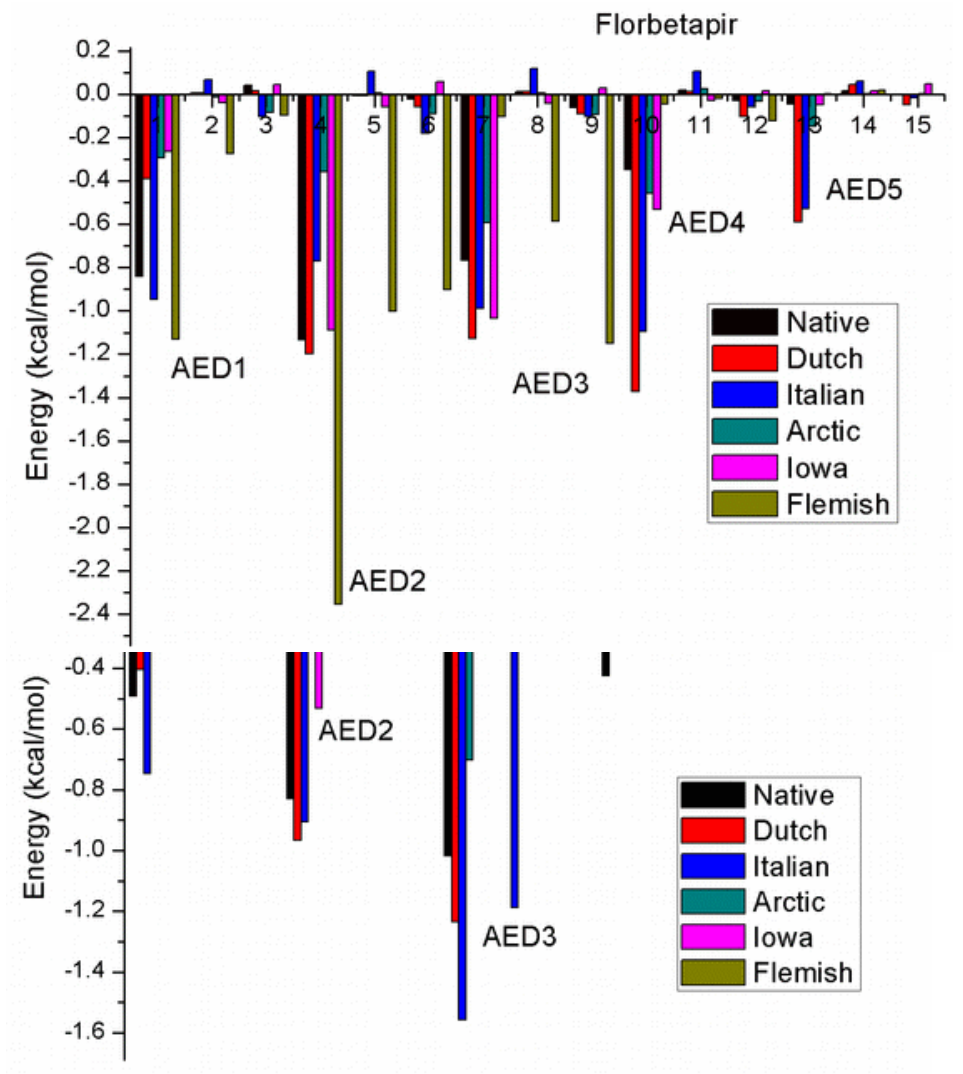

Figure 6 . The residue wise contribution of the mutated amino acid present in the $A \beta_{42}$ system towards the MM/GBSA binding free energy in the different $A \beta_{42}$-PET tracer systems. 


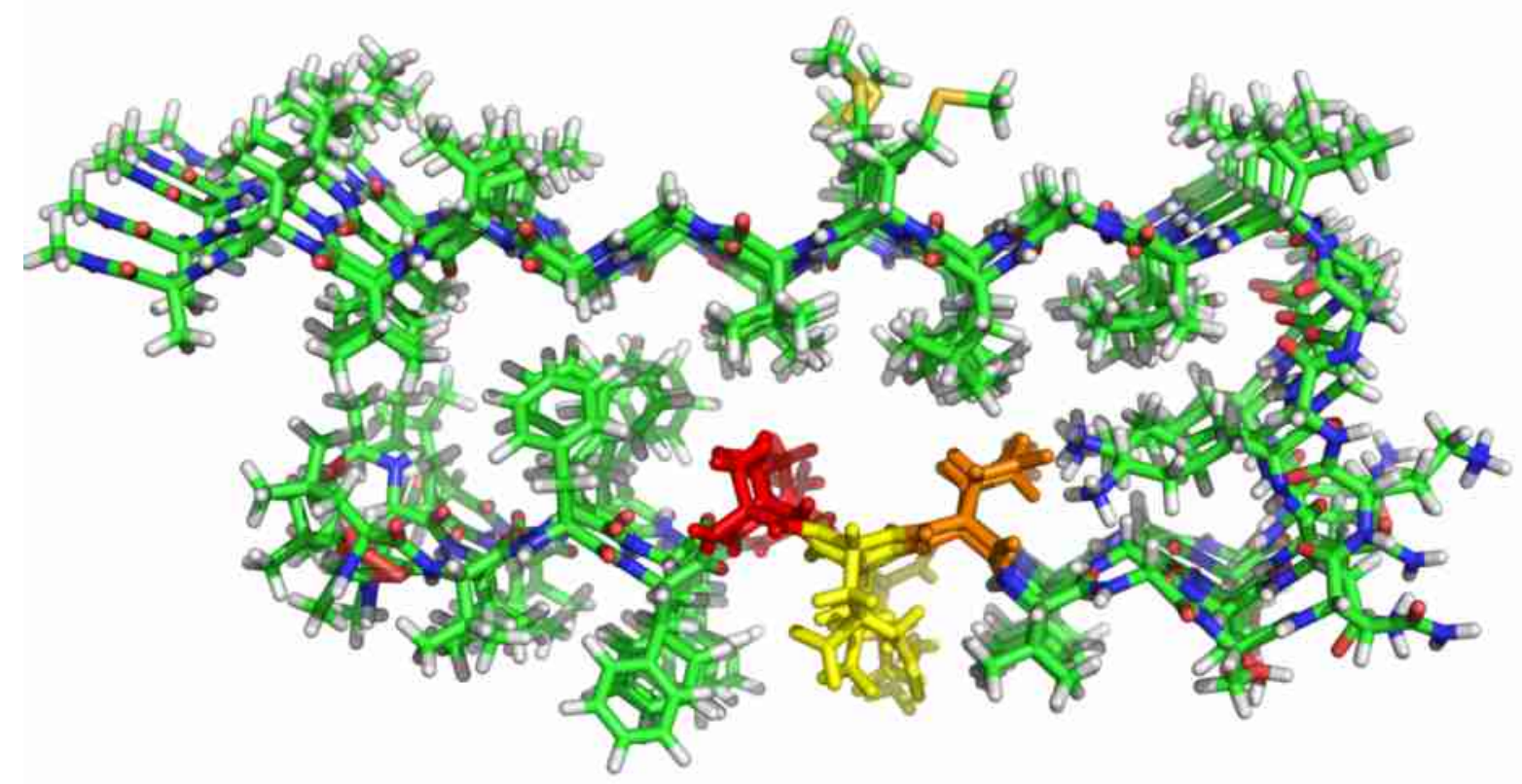

Figure 7. The $A \beta_{42}$ structure with the residue mutated highlighted (alanine 21(red), glutamic acid 22(yellow) and aspartic acid 23(orange)).

\begin{tabular}{|c|c|}
\hline${ }_{17} \mathrm{LVFF}_{\text {AEDVG SNKGA IIGLM VGGVV IA }}{ }_{42}$ & Native $A \beta_{42}$ \\
\hline${ }_{17} \mathrm{LVFF}$ AGDVG SNKGA IIGLM VGGVV IA ${ }_{42}$ & Arctic mutation \\
\hline${ }_{17} \mathrm{LVFF}_{\text {AKDVG SNKGA IIGLM VGGVV IA }}$ & Italian mutation \\
\hline${ }_{17} \mathrm{LVFF}$ AQDVG SNKGA IIGLM VGGVV IA ${ }_{42}$ & Dutch Mutation \\
\hline${ }_{17} \mathrm{LVFF}_{\mathrm{AENVG}}$ SNKGA IIGLM VGGVV IA ${ }_{42}$ & Iowa mutation \\
\hline${ }_{17} \mathrm{LVFF}$ GEDVG SNKGA IIGLM VGGVV IA 42 & Flemish \\
\hline
\end{tabular}

Scheme 1. Illustration of the amino acid sequences of different $A \beta_{42}$ system under investigation. 


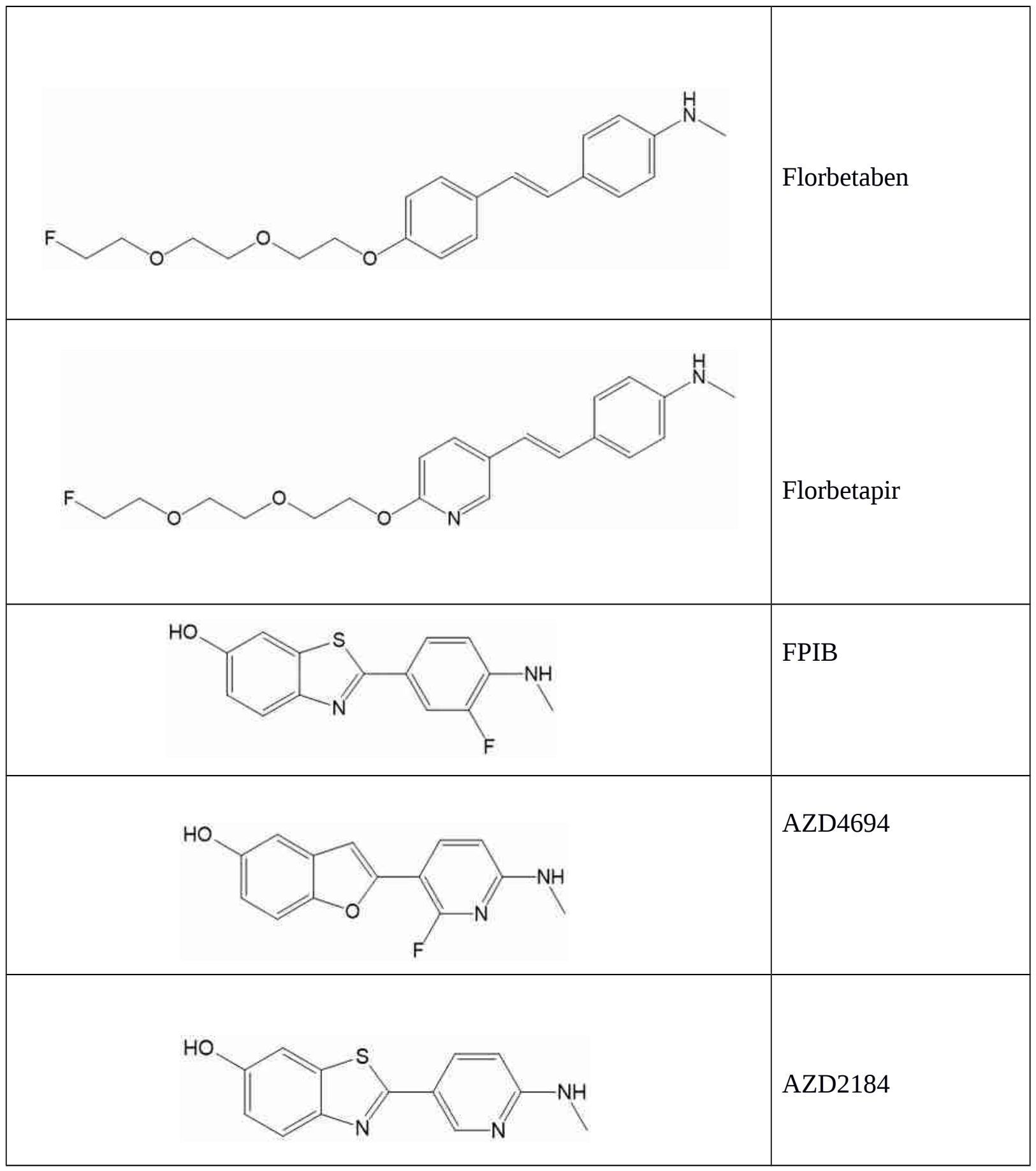

Figure 8. The structure of different PET tracers used in this study. 